\title{
Augmented Reality and Virtual Reality in Education. Myth or Reality?
}

\author{
https://doi.org/10.3991/ijet.v14i03.9289 \\ Noureddine Elmqaddem \\ Ecole des Sciences de l'information (ESI), Rabat, Morocco \\ nelmqaddem@esi.ac.ma
}

\begin{abstract}
Augmented Reality and Virtual Reality are not new technologies. But several constraints prevented their actual adoption. Recent technological progresses added to the proliferation of affordable hardware and software have made AR and VR more viable and desirable in many domains, including education; they have been relaunched with new promises previously unimaginable. The nature of AR and VR promises new teaching and learning models that better meet the needs of the 21 st century learner. We're now on a path to reinvent education. This work consists of explaining the reasons behind the new rise of AR and VR and why their actual adoption in education will be a reality in a near future.
\end{abstract}

Keywords-Augmented reality, Virtual reality, education, immersive learning

\section{Introduction}

Augmented reality and virtual reality are not really new technologies. The first virtual reality headset was created at the University of Utah in the 1970s by Daniel Vickers. With two screens, the headset gives the user the opportunity to observe the virtual scene presented to him by turning his head. A few years later, a new interface is developed: the data glove (DataGlove). This device, created in 1982, measures the movement of the hand and fingers and communicates it to the computer (Fuchs, 2006).

And, the term "Virtual Reality" was proposed in the United States in the 1980s by Jaron Lanier [1]. As for the term "augmented reality", it was coined by researcher Thomas Caudell and David Mizell in 1990 to describe how the head-mounted displays that electricians used when assembling complicated wiring harnesses worked.

The last technological craze of AR and VR was in the 1990s. But, at that time, many constraints prevented these technologies from being actually adopted by the general public.

In this work, we will argument in favor of a real advance of AR and VR and explain why they finally can be adopted in all domains, including education. We will explore the evolution of some technologies that are used in AR. Hence, we will try to verify whether AR and VR are a reality and their adoption in education is finally possible or are they still a myth. 


\section{Objective}

Explain why AR and VR can finally be seriously integrated into teaching and learning and demonstrate how much their promises are high.

\section{Questions}

There are 2 important questions behind this work:

- What makes AR and VR a reality no longer just a myth?

- Why are AR and VR a real new enhancement of teaching and learning? What makes them more suitable for the 21 st century student than the different methods of learning we have known until now?

\section{$4 \quad$ Research Method}

Comparison of some technologies used during the 1990s and their current counterparts that affected the use of AR and VR.

\section{$5 \quad$ Results}

The nature of AR and VR and their recent enhancement thanks to various technological advancements allows a new type of learning that better meets the needs of the 21 st century learner who wants entertainment, interactivity, participation and manipulation of objects. However, an effective adaption of AR and VR in education and learning will not happen until some technical and social issues are resolved and education programs are more adapted so as to take full advantage of the potential of these technologies.

\section{Discussion}

\subsection{Virtual reality}

A favorite definition, though restrictive, is to consider virtual reality as "human immersion in a synthetic world" [2]. This definition is especially acceptable for people who associate virtual reality with the use of a helmet. And, this is exactly the case that interests us in this work. Compared to the virtual reality experiments of the $90 \mathrm{~s}$, this technology has improved to an impressive degree.

Virtual reality (VR) is a technology that allows us to immerse ourselves in an artificial world; this world can be an entirely imaginary universe or only the reproduction 
of the real world. The experience can be visual, auditory and, at times, also haptic1. This immersion is done using a virtual reality headset that places a stereoscopic 3D display system in front of the eyes (on the nose). Some models are equipped with sensors that detect head tracking to allow the user to look around. The images are then recalculated in real time to synchronize with the direction of the head or gaze.

VR has sought to get the attention of the general public since the $1980 \mathrm{~s}$, but without an actual success. At that time, technologies related to VR were not known to the general public, but then they aroused the interest of the media.

Since 2014, virtual reality dedicated to the general public has been boosted by the arrival of helmets that are both more efficient and affordable. The developer version of the Oculus Rift headset was released in 2013, and it is only in March 2016 that this headset was on the mainstream market. Google was the first to play the card of the democratization of this technology by proposing in 2014 a model of VR helmets in cardboard called Google Cardboard and which is used with a smartphone as a display system. Subsequently, other companies have manufactured more advanced versions of the Cardboard, such as Samsung through its Gear VR (the mobile version of the Oculus Rift). Versions of VR headphones connected to a computer or game console are also available on the public market since the year 2016. Besides the Oculus Rift headphones from Facebook, there is HTC's HTC Vive and Sony's PlayStation VR amongst many others. These versions of high-end headsets are more expensive and require the use of a high-performance computer or a recent game console. Less expensive helmet projects that work with underperforming computers are in progress.

It is only from 2012 that virtual reality finally looks interesting. Several technological and commercial improvements now allow the use of VR with enough comfort to arouse the interest of everyone. On the one hand, the accessibility and power of computers and smartphones. On the other hand, the generalization of Internet access and the speed of fixed and mobile connections. Not to mention the richness and diversity of content in VR (thanks to the improvement of other technologies and programming languages). At the same time, picture or video quality has improved a lot. The "headtracking" also improved so much that the use of VR helmets is finally possible without discomfort.

An important evolution in the field of virtual reality is related to the possibility of manipulating the objects of the synthetic world through the use of controllers such as Oculus Touch. This allows students, for example, to practice and learn by interacting with objects in the virtual world in an even more interesting way.

According to Michael Abrash, head of the scientific team of the company Oculus VR, what the VR allows us to do today was technically almost impossible a few years ago. The evolution experienced by VR these last 5 years is certainly remarkable. But, what we will see in the next few years will be even more impressive. (Abrash, 2016)

Giants like Google, Facebook, HTC and Sony (through their Cardboard, Oculus Rift, HTC Vive and Playstation VR helmet) have given a new breath to this technology with promises of real success this time. They are working on gigantic projects to

\footnotetext{
${ }^{1}$ Allows us to experience certain sensations related to touch
} 
advance this technology and enable its exploitation in several areas, including the field of education.

According to the investment bank Citi, the hardware, software, networks and content in VR market will reach $\$ 200$ trillion in 2020.

The fields of application of this technology are unlimited: training with simulators, simulation of surgical procedures, architecture, archeology with the reconstruction of sites, virtual museum visits, treatment of phobias, and various types of learning.

Just like flight simulation, which has long been known for its effectiveness in learning to pilot when combined with actual flights, why not do the same thing in the field of education? Michael Bodekaer wants to do even better through his virtual lab that gives scientists, whether experienced or in training, the opportunity to do tests and experiments without taking physical risks, and with less costs and more results. There are also immersive education platforms, such as Engage, a free to use social education and presentation platform that allows everyone to hold meetings, classes, private lessons and presentations with people from all around the world in a safe virtual multi user environment. ${ }^{2}$

The value of adopting virtual reality in education and learning is related in part to the fact that this technology can improve and facilitate learning, increase memory capacity and make better decisions while working in entertaining and stimulating conditions. In fact, when we read textual content (on a printed document for example), our brain uses a process of interpretation of everything we read, which increases our cognitive efforts. In the case of the use of virtual reality, the process of interpretation is reduced because there are fewer symbols to interpret and the understanding is more direct. For example, it is easier to understand how a machine works by visualizing the process of its operation than by reading a textual explanation. And, when the visualization is in $3 \mathrm{D} / \mathrm{VR}$, it is even clearer. Having physical access to all what we learn is not possible, hence the importance of VR, which allows us to access everything we want, virtually, as if we really are there. The learner can for example explore the moon or the ocean floor or the state of a place as it has been in the past. This allows a better understanding of things and phenomena with less cognitive efforts on the part of the learner, and less cost for the institute that deals with learning. The learner feels more engaged, more motivated and more receptive and ready to learn and communicate with others. Virtual reality-based learning has been proven to increase learners' level of attention by $100 \%$ and improve test results by $30 \%$.

VR will not only transform the way we entertain ourselves, but it will also completely change the way students learn in and out of the classroom. However, it will be necessary to know how to build and deploy educational programs that are well adapted to this technology and that best meet the requirements of the learner of the 21 st century. (Education and VR: Changing the way we learn. https://youtu.be/SxyuCbMK rI).

\subsection{Augmented reality}

Unlike virtual reality (which leads to total immersion in the artificial world), augmented reality refers to a virtual interface, in $2 \mathrm{D}$ or $3 \mathrm{D}$, that enhances (or augments) 
what we see by overlaying additional information (digital content) onto the real world. Immersion in the virtual world is not total, because we can always see the real world around us.

AR works through a device that films the real world and inverts live virtual objects, animations, texts, data or sounds that the user views from the screen of a computer, a smartphone, a tablet, a pair of glasses, a headset or any other on-screen display system. The real world and virtual information are synchronized thanks to geolocalization and embedded sensors (accelerometer, gyroscope) that locate the user in relation to his environment and adapt the display to his movements.

Until 1999, AR was confined to scientific research labs. Equipment was expensive and software complicated enough to make the normal user unable to deal with such a technology. The release of ARToolKit ${ }^{2}$ to the open-source community by H. Kato and Billinghurst in 1999 gave a new start to that technology. At that time already, they proposed an augmented reality conferencing system, which uses the overlay of virtual images on the real world and allows users to collaboratively view and interact with virtual objects using a shared virtual whiteboard [3]. In recent years, AR has attracted the interest of several actors around the world: Total Immersion, Wikitude, Layar and many others. Since then, this technology has evolved and became widespread thanks to the accessibility of smartphones and tablets. The manufacture of AR glasses such as Microsoft's HoloLens has boosted interest in this technology. Promising projects are in progress and will allow the real exploitation of this technology in all areas, including the field of education.

Among the works done so far on how advanced technologies can be harnessed to enrich teaching, many see AR as an effective tool [4]. Numerous studies have revealed positive effect of VR on the learning process [5]. This technology makes it possible to interact with objects that belong to the virtual or real world, to learn through experimentation, participation and interactivity, to increase motivation and attention of the learner [6]. Learning becomes more enjoyable and effective, even when it comes to exploring and knowing abstract concepts or complex phenomena, and this thanks to the possibilities of visualization and realization of the concepts that this technology makes accessible to the learner [7], [8].

AR tools and applications, including those dedicated to the field of teaching and learning, are numerous and evolve quickly. Experts predict that in the near future, AR will be the new computing platform. Screen machines, such as computers and telephones, will be replaced by immersive devices based on VR and AR. The HoloLens augmented reality helmet from Microsoft has already gone in this direction. According to Pamela B. Davis (Dean, School of Medicine at Microsoft Build 2016), students will be allowed to learn using the most forward looking educational programs and HoloLens is a key part of this. It may be the next big transforming and change in medical education and many other fields. Oculus' John Carmack ascertains that the next 5 years will technologically and creatively take this medium some place we have never imagined ${ }^{3}[9]$.

\footnotetext{
${ }^{2}$ https://www.hitl.washington.edu/artoolkit/documentation/history.htm

${ }^{3}$ Oculus' John Carmack Explains Virtual Reality in 5 Levels of Difficulty

https://www.youtube.com/watch?v=akveRNY6Ulw
} 
The areas of application of AR affect entertainment (including video games) as well tourism, architecture, medicine, education, industry. In the sector of education and training, it allows technicians, for example, to learn new procedures in real conditions. Faced with a new device, the person can discover the disassembly procedure step by step by seeing the instructions appear in real time.

HoloLens for example allows medical students to manipulate and visualize the human body with unprecedented accuracy [10].

In the cultural field, augmented reality applications allow tourists or museum visitors to discover the history of places or works by simply pointing the camera of their smartphone in their direction.

According to Kurubacak and Altinpulluk [11], AR provides numerous educational benefits. For students, these benefits can be summarized as: courses' being fun, reducing cognitive load, increase in motivation and interest towards the course, increased opportunity to ask questions, increase in interaction between students, new opportunities for individual learning, concretizing abstract concepts, rise of success. As for teachers, these benefits consist of contribution to the development of creativity in students, ensuring effective participation of students to the course, students' being able to carry out the course with their own pace.

\subsection{Recent advances and new perspectives}

Advancements in AR and VR do not concern only hardware, but also software. For Oculus, Rift Core $2.0^{4}$ promises a more powerful, intuitive and personal VR platform. Rift Core 2.0 offers a brand new system interface, Oculus Dash and Oculus Home. Dash takes full advantage of touch and unlocks the entire power of computers allowing the manipulation of desktop apps from inside the VR environment. Hand presence is a powerful tool for direct manipulation. Thanks to Dash and Oculus Touch, VR as a computing platform will soon be no longer just a project, but a reality. Dash is meant to be a massive step forward for VR and will make it a fundamentally new computing platform, one we will use every day to work, connect and play.

On the other hand, Oculus Home and Facebook Spaces ${ }^{5}$ show that VR is not only for entertainment, but can be used for all our activities, including communication and socialization. This should attract the interest of most of those reticent people who argument against VR as simply an entertainment medium.

There are many AR platforms that allow developers, as well as beginners, to create augmented reality applications and products such as Augment ${ }^{6}$, Layar ${ }^{7}$ and Blippar ${ }^{8}$

\footnotetext{
4 Oculus announces Rift Core 2.0 within Oculus Connect 2017 launch event on October 112017. https://www.youtube.com/watch?v=fBjhAY1H35M. Thanks to Dash and Home, the Rift Core 2.0 update (expected on December 2017) allows a greater level of presence, interaction with friends and manipulation of applications within VR.

5 www.facebook.com/spaces

https://www.oculus.com/experiences/rift/1036793313023466/

https://www.facebook.com/FacebookTips/videos/10155260579068466/

6 www.augment.com

7 www.layar.com

8 www.blippar.com
} 
(amongst many others). As for Apple's and Google's developer's kits (ARKit ${ }^{9}$ and ARCore $^{10}$ respectively), they will simply open the door even wider for developers to enrich the AR content. Competition between Google and Apple through their AR platforms will make these technologies evolve quickly. ARKit and ARCore will allow developers to propose rich AR and VR contents and varied applications.

Accessibility of VR and AR is another element that should help to popularize these technologies. Google was the pioneer in proposing cheap VR platforms and headsets that started with Google cardboard in 2014 (The first VR platform and VR headset). In 2016, Google introduced a new VR platform and headset called Daydream (that costs less than 50 \$).

Accessibility of VR being very important, Oculus has been working on the project of new headsets that are more available. To profit from the best quality VR headsets like Oculus Rift, we had to own an expensive computer (that meets the needs of Oculus Rift). And Oculus Rift itself was proposed for $700 \$$. The Oculus Gear Vr too necessitates the new brand Samsung smartphones (Samsung S6, S7, S8 or Note).

Now, thanks to stand-alone headsets like the Oculus Go, we no longer need a computer nor a smartphone. Oculus Go allows a high quality VR experience and costs less about $200 \$$ for the $32 \mathrm{~GB}$ version and $250 \$$ for the $64 \mathrm{~GB}$ one. And the oculus Rift itself is more affordable (about 400\$). Apple is working on a AR glass called iGlass which will be released soon and should open new perspectives for AR. We also notice the accessibility of $360^{\circ}$ cameras that allow everybody to take photos or videos in VR $/ 360^{\circ}$. For $170 \$$, Samsung Gear 360 , for example, allows to capture high quality VR photos and videos (4K videos and 15 MP still images). Other VR cameras cost less than $100 \$$. Few additional improvements on these $360^{\circ}$ cameras will make the production of VR videos and photos accessible to everybody.

\section{Conclusion}

The main objective of this work was to verify to what extent AR and VR have evolved and whether they are now mature enough to be integrated in education programs. In fact, many recent hardware and software improvements show that in a near future AR and VR will be reliable enough as new computing platforms. This promises radical changes and new teaching and learning models that should satisfy the needs of the learner of the $21^{\text {st }}$ century who no longer thinks the same way as in the $20^{\text {th }}$ or $19^{\text {th }}$ century. The fact that giants like Facebook, Google, Microsoft and Apple consider VR and AR as interesting fields for investment promises a bright future for these technologies.

It's clear that an actual integration of these technologies requires lots of improvements and changes not only on the part of engineers and experts of AR and VR, but also teachers and all persons related to the field of educational. Engineers must pro-

\footnotetext{
9 ARKit is a new framework introduced within iOS 11 and that allows to easily create augmented reality experiences for iPhone and iPad. https://developer.apple.com/arkit/

10 ARCore is an augmented reality software development kit for Android released by Google on August 292017
} 
pose VR and AR headsets that are more comfortable and accessible. In fact, a long use of the VR and AR headsets proposed for now causes some discomfort. As for persons related to education, they must deploy more forward educational programs that fit well with the nature of these technologies and fulfil the needs of the learner. When applied properly, these technologies can create enhanced contemporary educational environments and enriched learning opportunities for students. In all cases, it is certain that in the next few years AR and VR will revolutionize the way we interact with the real world and will be widely adopted in all domains. They will no longer be only a myth but a reality. What has been said till now about these technologies does not show their actual potential. It is just scratching the surface of what they will allow us to do in the near future.

\section{References}

[1] P. Fuchs, G. Moreau and P. Guitton, Virtual Reality: Concepts and Technologies, Boca Raton: CRC Press, Inc., 2011.

[2] R. J. Seidel and P. R. Chatelier, Virtual Reality, Training's Future?: Perspectives on Virtual Reality and Related Emerging Technologies, Berlin: Springer Science \& Business Media, 1997. https://doi.org/10.1007/978-1-4899-0038-8

[3] H. Kato and M. Billinghurst, "Marker Tracking and HMD Calibration for a video-based Augmented Reality Conferencing System," in Proceedings of the 2nd International Workshop on Augmented Reality (IWAR 99), San Francisco, USA, 1999. https://doi.org/10.1109/IWAR.1999.803809

[4] M. Dunleavy, C. Dede and R. Mitchell, "Affordances and limitations of immersive participatory augmented reality simulations for teaching and learning," Journal of Science Education and Technology, vol. 18, no. 1, pp. 7-22., 2009. https://doi.org/10.1007/s10956$\underline{008-9119-1}$

[5] H. Kaufmann and M. Papp, "Learning objects for education with augmented reality," in Proc. of EDEN (European Distance and E-Learning Network) Conference, Budapest, Hungry, 2006.

[6] S. Singhal, S. Bagga, P. Goyal and V. Saxena, "Augmented chemistry: Interactive education system," International Journal of Computer Applications, vol. 49, no. 15, pp. 1$5,2012$.

[7] E. Klopfer and K. Squire, "Environmental detectives: The development of an augmented reality platform for environmental simulations," Educational Technology Research and Development, vol. 56, no. 2, pp. 203-228, 2008. https://doi.org/10.1007/s11423-007-90376

[8] D. Sumadio and R. D. R. A., "Preliminary Evaluation on User Acceptance of the Augmented Reality use for Education," in Second International Conference on Computer Engineering and Applications, Bali Island, Indonesia, 2010.

[9] J. Carmack, "Virtual Reality Engineer Explains One Concept in 5 Levels of Difficulty | WIRED," [Online]. Available: https://www.youtube.com/watch?v=akveRNY6Ulw. [Accessed 1305 2018].

[10] M. Griswold, "Hololens in Medicine: amazing demo from Microsoft Build 2016 YouTube," 2016. [Online]. Available: https://youtube/GBs471Ki8HE. [Accessed 1806 2018]. 
[11] G. Kurubacak and H. Altinpulluk, Mobile Technologies and Augmented Reality in Open Education, I. S. Reference, Ed., Hershey, 2017. https://doi.org/10.4018/978-1-5225-2110-5

[12] M. Abrash, "Oculus Connect 3 Opening Keynote: Michael Abrash," 2016. [Online]. Available: https://youtube/AtyE5qOB4gw. [Accessed 1106 2018].

\section{$9 \quad$ Author}

Noureddine Elmqaddem is associated with Department of Document Engineering, Strategic Intelligence and Records Management (IDVSRM) at Ecole des Sciences de l'information (ESI) in Rabat, Morocco.

Article submitted 28 July 2018. Resubmitted 22 October 2018. Final acceptance 24 October 2018. Final version published as submitted by the authors. 